\title{
Recent Developments in Clean up Techniques of Pesticide Residue Analysis for Toxicology Study: A Critical Review
}

\author{
Shaon Kumar Das \\ ICAR Research Complex for NEH Region, Sikkim Centre, Gangtok, Sikkim, India \\ *Corresponding Author: shaon.iari@gmail.com
}

Copyright $(2014$ Horizon Research Publishing all rights reserved.

\begin{abstract}
Clean up is one of the most important steps to remove co-extractives and undesired interfering substances from the sample before analysis in pesticide residue analysis. Some advanced, sophisticated and efficient methods of clean up have emerged out which is able to save time as well as amount of solvents. These are Solid Phase Extraction (SPE), Gel Permeation Chromatography (GPC) and Supercritical Fluid Chromatography (SFC). They are very much efficient for clean up and extraction of pesticide samples. SPE method is successfully used for clean up of pesticide residue from soil and water samples. GPC is now widely used in sample preparation in many residue laboratories and is effectively used for multi-residue analysis. SFC is by far the best method of clean up and extraction, though it involves high cost. Thus, various suitable clean up methods can free pesticide residues from extraneous co-extractive materials in an efficient manner and helps to prepare a good sample for further analysis.
\end{abstract}

Keywords Clean up, Co-Extractives, Solid Phase Extraction, Gel Permeation Chromatography, Super Critical Fluid Extraction

\section{Introduction}

Pesticides are chemicals that are used in agriculture for control of pests, weeds and plant diseases. Pesticides are used both in agriculture and as vector control agents in public health programmes. In living organisms, pesticide can be stored in fat tissues, where they accumulate and increases over time [7]. While extracting the pesticide with solvents from the plant materials, proteins, tannins, lipids, fat, waxes, chlorophyll and terpenoids, they are co-extracted from matrix of substances [5]. These co-extractives can prevent the reaction of pesticides with chromogenic reagents, coloured extracts directly interferes in the colorimetric analysis and can also contaminate the columns and detectors in the analysis. There are four logical approaches in the clean up requisite to the final determination like: when the insecticide is evaluated by direct measurement (only superficial cleanup is necessary), when the insecticide can be measured only after all interfering substances have been removed or converted into non interfering products, when insecticide can be measured after having converted to suitable derivative and then isolated and when methods of compensation can be used [9].

To achieve necessary sensitivity, the interfering substances have to be removed from pesticide, and this step is known as "clean up". The type and extent of clean up will depend on final method of pesticide analysis [18]. Clean up is required when pesticide has to be measured after having converted to a suitable derivatives and then isolated.

\section{Commonly Used Methods in Clean up}

\subsection{Steam Distillation}

To remove natural oils, fats or other steam volatile co extractives from steam non volatile pesticides. Sometimes steam volatile pesticides (Aldrin, Chlordane, Lindane) are removed from non volatile extractives [2].

\subsection{Partition}

Polar pesticides can be cleaned up from the non polar extracts by partitioning with polar solvents. The conversion is also true. Pigments and waxes are preferentially soluble in n-hexane, and pesticides because their high polarities gets extracted in acetonitrile which can be evaporated or diluted with water and re-extracted with hexane to recover pesticides [10].

\subsection{Sweep Co-Distillation}

Substrate extractives are injected in a glass column packed with glass wool, followed by repeated injection of the solvent. $\mathrm{N}_{2}$ gas sweeps the volatile compounds along with pesticide. Interfering substances remain in column while the pesticide elutes out.

\subsection{Coagulation}


It is used for cleanup of carbamate pesticide only. Aqueous solution of $\mathrm{NH}_{4} \mathrm{Cl}$ and phosphoric acid is added to the filtered solution to precipitate interfering substances which are then removed by filtration.

\subsection{Oxidation}

To determine organophosphorus pesticides, they are oxidized to inorganic phosphate by mixture of fuming nitric acid and perchloric acid. Inorganic phosphate is determined by molybdenum blue method [8].

\subsection{Saponification}

Fats, oils and triglyceride containing samples can be easily cleaned by refluxing with alcoholic $\mathrm{KOH}$ to convert triglycerides to soaps and glycerine.

\subsection{Adsorption Chromatography}

Using florisil, $\mathrm{MgO}$, alumina and celite, sometimes mixtures of adsorbants are also employed. Polythylene coated alumina is probably effective in both adsorption and absorption processes for removal of liquid and other interfering substances.

\subsection{Disadvantages}

Several disadvantages are there in traditional methods like: the methods are time consuming, larger sample handling,less recoveries,less accuracies,less safety to workers andless automation and also manual faults may creep in.

\section{Column Clean up}

This method is used in multi-residue methods on vegetables with 52 pesticides.Extracted pesticide fractions are generally divided into two parts.At first fraction, two samples were loaded on a silica gel mini column and the elute was collected in a $100 \mathrm{ml}$ RB flask.Florisil mini column is inserted on the silica-gel micro column, fraction one is loaded and eluted with 15 ml acetone:pet-ether(3:7).Combined elute is concentrated and injected to GC. Recovery of this method is $82 \%-104 \%$ depending on pesticides [17]. But it is also not advanced and sometimes co-extractives are also present in the sample.

\section{Solid Phase Extraction (SPE) and Clean up}

It is an extraction method using a solid phase and a liquid phase to isolate one, or other type of analyte from a solution. It is usually used to clean up a sample before using a chromatographic or other analytical method. The principle of action is based on simple adsorption chromatography where the mobile phase moves through the stationary phase and gets separated based on their respective $\mathrm{K}$ value [13].

\subsection{Benefit Solid Phase Extraction}

SPE is used in laboratories for various reasons like: faster sample preparation- average time cut by $2 / 3$, lower cost-less solvent and reagent consumption means less hazardous waste, less sample handling- no emulsion problems, easy automation, simultaneous batch processing of multi samples, greater recoveries- minimal sample transfer, greater accuracy, no cross contamination, improved safety-due to less solvent sample handling. In SPE, at first sample is prepared by homogenization, suspension, centrifugation etc [13]. Then weakly retained substances are washed off by weak solvents and sample is loaded onto conditioned cartridge. Then typically performed by loading the complex sample onto a preconditioned extraction cartridge containing a chromatographic sorbent and finally product is eluted with strong solvent and analyzed by HPLC, GC. Here, $3 \mathrm{ml}$ cartridge on a vacuum manifold is used, which increases the solvent flow rate through the cartridge. A collection tube is also placed beneath the SPE cartridge to collect the liquid that passes through the column.

Table 1. Chromatographic modes and sorbent types used in SPE

\begin{tabular}{|c|c|c|c|}
\hline Properties & Normal Phase & Reverse Phase & Ion Exchange \\
\hline $\begin{array}{c}\text { Sorbent } \\
\text { polarity }\end{array}$ & High & Low & High \\
\hline $\begin{array}{c}\text { Typical } \\
\text { solvent } \\
\text { polarity range }\end{array}$ & $\begin{array}{c}\text { Low to } \\
\text { medium }\end{array}$ & $\begin{array}{c}\text { High to } \\
\text { medium }\end{array}$ & High \\
\hline $\begin{array}{c}\text { Typical } \\
\text { sample }\end{array}$ & $\begin{array}{c}\text { Hexane, } \\
\text { Tolune, } \\
\text { DCM }\end{array}$ & $\mathrm{H}_{2} \mathrm{O}, \mathrm{Buffers}$ & $\mathrm{H}_{2} \mathrm{O}, \mathrm{Buffers}$ \\
\hline $\begin{array}{c}\text { Elution } \\
\text { solvents }\end{array}$ & $\begin{array}{c}\text { Ethyl acetate, } \\
\text { acetone, } \\
\text { CH }\end{array}$ & $\begin{array}{c}\mathrm{H}_{2} \mathrm{O}, \mathrm{CH}_{3} \mathrm{CN}, \\
\mathrm{CH}_{3} \mathrm{OH}\end{array}$ & Buffers,salts \\
\hline $\begin{array}{c}\text { Solvent } \\
\text { Change }\end{array}$ & $\begin{array}{c}\text { Increase } \\
\text { solvent } \\
\text { polarity }\end{array}$ & $\begin{array}{c}\text { Decrease } \\
\text { solvent polarity }\end{array}$ & $\begin{array}{c}\text { Increase ion } \\
\text { strength or } \mathrm{pH}\end{array}$ \\
\hline $\begin{array}{c}\text { Sample } \\
\text { elution }\end{array}$ & $\begin{array}{c}\text { Least polar } \\
\text { component } \\
\text { first }\end{array}$ & $\begin{array}{c}\text { Most polar } \\
\text { component first }\end{array}$ & Weakly ionized \\
\hline
\end{tabular}

\subsection{Disadvantages of SPE}

Some problems of SPE are incomplete removal of interferences due to similar behavior of interferences and analytes; low recovery due to adsorption of analyte to matrix, incomplete elution, mass overload, poor conditioning; high variability due to method variability, operator variability [11]. But main disadvantages are unproven for many pesticides, unable to handle large sample sizes, generally are ineffective for extracting water-soluble pesticides and metabolites.

\section{Gel Permeation Chromatography}


Also known as Size Exclusion Chromatography (SEC), it is a chromatographic method in which molecules are separated based on their size. It is widely used in the analysis of polymer molecular weights (or molar mass). The extraction process for determining semi volatile organic compounds in environmental samples extracts the compounds of interest along with a number of other constituents. These other constituents are detrimental to the performance of the analytical method, causing deposits in the injectors of GC and GCMS instrumentation, leading to erratic results [1]. Lipids in tissue samples and soils with high levels of organic matter Gel Permeation Chromatography is very well-suited for removal of these compounds. GPC is a technique which separates components of a sample based on their molecular size. Since lipids are very large molecules compared to the target compounds in these methods, they are effectively removed from the extract prior to analysis [3].

\subsection{Principles of Operation}

When a sample is injected into the column, the polymer molecules are separated according to their hydrodynamic volumes. GPC removes fats and other large bio-molecules from sample extracts.Polymer molecules larger than the pores of the packing material cannot enter the pores and are eluted at the interstitial volume $\mathrm{V}_{\mathrm{i}}$. Small molecules, however, have access to the pores and will therefore elute at the sum of both interstitial and pore volume, i.e. $V_{i}+V_{p}$. Molecules which have sizes between the above two extreme values will have access to only a part of the pore volume and will therefore be eluted at an elution volume $V_{e}$ which is given by-

$\mathbf{V}_{\mathbf{e}}=\mathbf{V}_{\mathbf{i}}+\mathbf{K}_{\text {SEC }} \mathbf{V}_{\mathbf{p}}$ where $\mathrm{K}_{\text {SEC }}$ is the equilibrium constant of a sample in size exclusion chromatography $\left(0<\mathrm{K}_{\mathrm{SEC}}<1\right)$.

\subsection{Consideration}

For GPC work to be reproducible there must be control over flow rate, temperature, solvent composition, tunnel size of the stationary particles (it must not change), the sample polymer must be soluble in a suitable solvent, the solvent must be filtered to dissolve the sample before running it through the GPC.

\subsection{GPC Column Preparation}

A $50 \mathrm{~g}$ of the packing material Bio-BeadsSX-3 (Bio-Rads, Richmond, USA) -is suspended in $100 \mathrm{ml}$ elution solvent for $24 \mathrm{hr}$. The gel is then transferred into a vacuum flask and degassed for $5 \mathrm{~min}$. The slurry is packed into the GPC column and the plunger is adjusted (or depressed) to give a bed height approximately $20-35 \mathrm{~cm} \mathrm{[3].}$

\subsection{GPC Clean-up}

The column is eluted with the GPC elution solvents for 30 min. Following this, $10.0 \mathrm{ml}$ of the $0.08 \mu \mathrm{g} \mathrm{ml}^{-1}$ pesticide-oil mixtures is injected into the Rheodyne injector using a syringe fitted with a PTFE filter. A $5.0 \mathrm{ml}$ of the mixture are injected into the column (the remaining $5.0 \mathrm{ml}$ being diverted into a waste flask). The elution solvent is pumped through the column at a constant flow rate of $2.0 \mathrm{ml} \mathrm{min}{ }^{-1}$. A $5.0 \mathrm{ml}$ of the clean-up eluate are collected in a $250 \mathrm{ml}$ round bottomed flask [15]. The solvent is evaporated to $5.0 \mathrm{ml}$ and $1.0 \mu \mathrm{g}$ of the concentrated eluate is injected into the gas chromatograph for determination. The remaining pesticide-oil mixtures were analysed in a similar manner.

\subsection{Advantages of Using GPC as Cleanup Technique}

The main advantage of GPC is it improves the method performance,

- extends GC column life, renders more efficient analyses, requires only the final extracts to be concentrated down, facilitating auto sampling automatic fraction collection as part of the GPC system, cleanups can be performed with little or no operator intervention, no pesticide loss at columns, GPC followed by SPE gives better results. It is done to determine organochlorine pesticides in eggs.

\subsection{Disadvantages}

A medium-pressure piston type pump is required to deliver solvent to the column, making a sample injection valve necessary.

The required equipment is more expensive than that used in adsorption chromatography, although such equipment is available in an automated package.

\section{Supercritical Fluid Chromatography (SFC)}

SFC is a unit operation to exploit high dissolving power of fluids at temperature and pressure above critical values for extracting analytes from sample matrixes.

Liquids chromatography is fairly readily adapted to industrial scale because sufficient feedstock can be passed through the chromatograph. On the other hand gas phase chromatography has a role primarily in the lab, because gas densities are 1000 times lower than the corresponding liquid phase. Supercritical Fluids lie in the middle ground, so there is hope to use some gas phase properties imparted to a high density solute. Supercritical Extraction has led the way in developing separation techniques with supercritical fluids. Some success has been reported with laboratory research in the area of Supercritical Fluid Chromatography (SFC) and there seems sufficient incentive to think about industrial processing - perhaps even continuous industrial processing. $\mathrm{CO}_{2}$ is the most common supercritical solvent and the one for which we have the most information [16]. As an example of 
the dramatic effect of the property changes in the critical region solvent for no polar molecules like naphthalene, but performs less well with polar fluids.Beside from $\mathrm{CO}_{2}$, there are several other compounds that are commonly considered for use as supercritical solvents, the most common of which are given in the accompanying table.

\subsection{Supercritical Extraction}

For over a quarter of a century supercritical fluids, primarily carbon dioxide, have been used as a solvent in extraction processes performed under supercritical conditions. Carbon Dioxide has several properties that recommend it for this duty. Its critical temperature is $31.3^{\circ} \mathrm{C}$, making near room temperature operations possible. It is non-toxic, non-flammable and approved by FDA for use in food and pharmaceutical plants. Its critical pressure is 72.9 atm., which is considered moderate. Its properties have been exhaustively studied, so it continues to be the extraction solvent of choice. Caffeine removal is an excellent example of the application of this technology. A sample is placed in the Sample thimble, and supercritical fluid is pumped through the thimble. The extraction of the soluble compounds is allowed to take place as the supercritical fluid passes into a collection trap through a restricting nozzle. The fluid is vented in the collection trap, allowing the solvent to either escape or be recompressed for future use. The material left behind in the collection trap is the product of the extraction. Obviously this is a batch process. This is acceptable for the analytical purpose to which the method is applied, but could not be considered commercially, unless there was some extreme purpose to which the process was being applied. The extraction efficiency, as we have shown earlier, temperature and pressure are important means of affecting solubility. For instance raising the pressure often increases solubility. Obviously changing the solvent will provide different solvent properties. Supercritical water will give radically different solubilities from ethylene or carbon dioxide [19]. Anhydrous ammonia will give still other results. Adding a small amount of a second material (co solvent) to the solvent, such as a few percent methanol to carbon dioxide, enhances the solubility of hydrophilic compounds. One example of supercritical extraction, consider that we place a soil sample in the thimble, and perform the analytical extraction at different pressures. The analysis of interest in this case is 2,4,5-T -trichlorphenyloxyacetic acid. It is evident in this case that we get a far greater extraction of the pesticide from soil at the higher pressure, all other things being equal [4]. The recovery of the pesticide varies linearly with increase in pressure. At 300 atm we can expect an $80 \%$ recovery. For analytical purposes a strong signal and a good calibration curve are more important than maximizing recovery.

\subsection{Advantages of SCF Clean Up}

Fast extraction rate: Low viscosities allow the solvent to penetrate the pores of the solid matrix easily and high diffusion coefficients of solute allow rapid mass transfer of solute out of the matrix. $\mathrm{CO}_{2}$ is typically used as $\mathrm{SC}$ fluid as it is non toxic, leaves no residues, no disposal costs and less expensive than liquid solvents. Collection of analyte is easy by reducing the extraction solvent pressure to atmosphere and letting the solvent to vaporize.

- Heat labile compounds remain unchanged.

- No environmental risk and fire hazards

- By simply changing the pressure of SCF selectivity of extraction can be controlled and changed.

\subsection{Factors Affecting Extractions}

In developing a unified screening and quantification method priority must be given to both speed and good pesticide recovery without matrix contamination. To fulfil these requirements SFE optimisation must encompass extraction as well as trapping and elution of the compounds of interest. Considering fat contamination two lipid fractions can be distinguished. One fraction of lipid is readily extracted using either organic solvent or SFE, whereas total extraction including phosphorylated and more polar lipids requires a more harsh treatment, such as boiling in hydrochloric acid prior to extraction. Thus the latter lipid fraction is less likely to contaminate residue extracts wherefore optimising SFE of OPPs from fatty matrices implies extraction of pesticides separately from the more accessible lipids. Addressing selectivity, the extraction characteristics of both pesticide and fat needs to be established [6]. As for the process underlying SFE, several authors have provided theoretical descriptions. From such studies, it has been found that several extraction parameters such as temperature, density, extraction time, and flow rate exert a strong influence on extractable analyte recoveries. In the present study a reduced factorial design was employed to select the major factors with respect to extraction efficiency. The four factors evaluated were supercritical fluid temperature, density, flow rate, and dynamic extraction time. It was observed that all factors had significant effect upon fat extraction, flow rate being the least significant factor. In contrast, only density and temperature had significant effects on recovery for the pesticides investigated (confidence level $95 \%$ ). In the present study only moderate flow rates were investigated. A more significant effect on residue recovery could be anticipated when using high flow rates due to low trapping efficiency associated with a high gas flow through the trap. As the supercritical fluid density is defined by temperature and pressure optimisation strategies may involve only one of these parameters. However, it has been observed that in optimising SFE methods it is of importance to vary both temperature and density, possibly in combination [19]. This is based upon the observation that the solubility of an analyte in a supercritical fluid is influenced by both the volatility of the analyte and the solvating effect of the supercritical fluid. The present study confirms the need for optimising temperature and density in combination 
and the experimental approach was to characterize the combined effect of temperature and density through a series of recovery experiment. The SFE method presented is substantially more environmentally favourable due to the very low solvent requirement, i.e., less than $20 \mathrm{ml}$ per sample including system cleaning. Additionally, total residue analysis ranging from raw meat sample to fully quantified results can be obtained within hours. In contrast, when analyzing an identical sample using an organic solvent extraction method the results will not be available until next laboratory day due to comprised labour intensive procedures. Thus the SFE method presented constitutes a promising alternative for fast routine residue analysis in fatty matrices such as meat.

\subsection{Disadvantage}

The total clean-up process is very expensive so cannot be affordable at all levels where highly efficient clean up is not so much needed.

\section{Conclusions}

Advanced techniques of clean up are more sophisticated and efficient than the traditional ones. Of the three, by far, Super Critical Fluid clean up is the most efficient one, though expensive. In the advanced clean ups GPC is one of the widely used clean up methods this days. For small amount of sample load SPE can be very good method of clean up-both in single and multi steps.

\section{REFERENCES}

[1] Anonymous, 1991. Method FSCL Pest-1, Ministry of Agriculture, Fisheries and Food. Norwich, UK.

[2] Bowman MC, Beroza M, 1965. Extraction p-values of pesticides and related components in six binary solvent systems. J. Assoc. off. Anal Chem. 48: 943-952.

[3] Cunha SC, Fernandes JO, 2011. Multipesticide residue analysis in maize combining acetonitrile-based extraction with dispersive liquid-liquid micro-extraction followed by gas chromatography-mass spectrometry. Journal of Chromatography A, 1218, 7748-7757.

[4] DINOCAP (087), www.fao.org/ag/AGP/AGPP/Pesticid/JM PR/Download/98_eva/ dinocap.pdf, accessed on April 4, 2009.

[5] Erwin WR, Schiller D, Hoskins WM, 1955. Pesticide residue analysis, preassay purification of tissue extracts by wax column. J. Agric. Fd Chem. 3(8): 676-679.

[6] European Commission Regulation, 2012. (EC) No. 149/2008 (2012). Off. J. Eur. Union L 58/1-L 58/398.

[7] FAO, 1986. Guide to codex recommendations concerning pesticide residues. Part 1: General notes and guidelines. Food and Agricultural Organization, Rome, Italy.

[8] Faucheux LJ, 1968. Rapid clean-up for carbaryl, using channel layer chromatography. J. Assoc. off Anal. Chem. 51: 676-678.

[9] Handa SK, Agnihotri NP, Kulshrestha G, 1999. Pesticide residues: Significance, Management and Analysis. Research Periodicals and Book Publishing House, New Delhi.

[10] Hoskins WM, Erwin WR, Miskus, Raymond, Thornburg WW, Werum LN, 1958. Pesticide residue analysis, A polyethylene-alumina column for purification of tissue extracts before analysis. J. Agric. Fd. Chem. 6(12): 914-916.

[11] Kalra RL, 1980. Clean-up procedures for insecticides residue analysis. In: Residue analysis of insecticide (ed. Gupta DS,), Department of Entomology, HAU, Hisar 40-56.

[12] Kumari, Beena, Kumar R, Kathpal TS, 2001. An improved multiresidue procedure for determination of 30 pesticides in vegetables. Pestic. Res. J. 13(1): 32-35.

[13] Kumari, Beena, Madan VK, Kumar R, Kathpal TS, 2002. Monitoring of seasonal vegetables for pesticide residues. Environ. Monit. And Assess. 74: 263-270.

[14] Method validation \& quality control procedures for pesticide residues analysis in food \& feed, 2011. Document No. SANCO/12495/2011.

[15] Oliveiraa MLG, Madureiraa F D, Aurélioa F, Ponteloa AP, Silvaa G, Oliveiraa R, 2012. A multi-residue method for the determination of pesticides in high water content matrices by gas chromatography-single quadrupole mass spectrometry with electron ionisation (EI-GC/MS). Food Additives \&Contaminants: Part A, 29, 657-664.

[16] Savant RH, Banerjee K, Utture SC, Patil SH, Dasgupta S, Ghaste MS, 2010. Multiresidue analysis of 50 pesticides in grapes, pomegranate and mango by gas chromatography-ion trap mass spectrometry. Journal of Agricultural and Food Chemistry, 58, 1447-1454.

[17] Sharma KK, 2007. Pesticide Residue Analysis Method. Directorate of Information and Publications of Agriculture, New Delhi: 6-10.

[18] Their HP, 1997. Harmonization of pesticide residue analytical methods by the Technical Committee, 275 of CEN. Pestic. Sci. 50: 151-55.

[19] Wu G, Bao X, Zhao S, Wu J, Han A, Ye Q, 2011. Analysis of multi-pesticide residues in the foods of animal origin by $\mathrm{GC}-$ MS coupled with accelerated solvent extraction and gel permeation chromatography cleanup. Food Chemistry, 126, 646-654 